\title{
Suicidal Risk in Older Patients with Depression During COVID-19 Pandemic: a Case-Control Study
}

\author{
LLC Louie, WC Chan, CPW Cheng
}

\begin{abstract}
Objectives: To compare older adults with late-life depression (LLD) and healthy controls in terms of suicidal ideation during the COVID-19 pandemic, and to determine predictors of suicidal ideation.

Methods: Between March and April 2020, old adults diagnosed with major depressive disorder (single or recurrent episode) as defined by the DSM-5 were recruited from psychiatric clinics or inpatient wards, whereas 31 healthy older adults without a history of depression or other psychiatric illnesses were recruited from voluntary organisations or elderly community centres. Their depressive symptoms, perceived severity of the pandemic, perceived time spent on receiving related information, perceived health, levels of loneliness, perceived coping efficacy, suicidal ideation, and the level of symptomatic responses to a specific traumatic stressor in the past week were assessed.

Results: In total, 21 men and 43 women aged 61 to 89 years were interviewed through telephone by trained research assistants. Of them, 33 were older adults with LLD (cases) and 31 were healthy older adults (controls). Older people with LLD had a higher level of suicidal ideation than healthy controls, after controlling for the level of depression and medical comorbidity $(F(1,59)=5.72, p=0.020)$. Regression analyses showed that coping efficacy and loneliness accounted for a significant portion of the variance in suicidal ideation, and loneliness significantly predicted the level of stress. Mediation analyses reveal an indirect effect between group and suicidal ideation through coping efficacy $(Z=2.43, p=0.015)$.

Conclusions: Older people with LLD are at increased suicidal risk and require timely mental health support. Coping efficacy and loneliness are important predictors for suicidal ideation and stress.
\end{abstract}

Key words: Aged; COVID-19; Depression; Suicide

Larissa LC Louie, Department of Psychiatry, The University of Hong Kong, Hong Kong

Wai-Chi Chan, Department of Psychiatry, The University of Hong Kong, Hong Kong

Calvin PW Cheng, Department of Psychiatry, The University of Hong Kong, Hong Kong

Address for correspondence: $\mathrm{Dr}$ Calvin Pak-Wing Cheng, Department of Psychiatry, 2/F, New Clinical Building, Queen Mary Hospital, 102 Pok Fu Lam Road, Pok Fu Lam, Hong Kong.

Email: chengpsy@hku.hk

Submitted: 17 June 2020; Accepted: 7 September 2020

\section{Introduction}

The coronavirus disease 2019 (COVID-19) pandemic has substantial effects on mental health and the suicide rate. ${ }^{1}$ Social-distancing measures (such as closing schools, work from home, and banning people from gathering in public places), self-isolation, and fear may exacerbate the adverse effects on mental health, ${ }^{2,3}$ especially among older people. During the 2003 severe acute respiratory syndrome (SARS) epidemic, there was a spike in the suicide rate among older people in Hong Kong, with a $31.7 \%$ increase compared with the previous year. ${ }^{4,5}$ Disconnectedness and fear of contracting SARS were more prevalent in older people with SARS-related suicide. ${ }^{2,6}$ Social-distancing measures disrupted the mental wellness of older people; some felt that they would create additional troubles for their families and resulted in loneliness and isolation. ${ }^{2}$

During a pandemic, people may exhibit traumatic stress symptoms such as having nightmares and intrusive thoughts. ${ }^{7}$ Higher stress scores from life events along with higher psychological distress are predictors of suicidal risk, and level of stress has a moderate association with suicidal ideation. ${ }^{8}$ People aged $>60$ years have higher COVID-19 peritraumatic distress scores and are more likely to be psychologically impacted by the COVID-19 pandemic. ${ }^{9}$

Patients with existing psychiatric disorders (eg, depression) are more likely than healthy individuals to experience and emotional response to the COVID-19 pandemic, resulting in relapse or worsening of symptoms. ${ }^{3}$ People with primary mood disorder diagnosis have significantly higher scores of COVID-19-related traumatic stress symptoms than those with no current mental disorder. ${ }^{10}$ In Hong Kong, around $10 \%$ of community dwellers aged $\geq 60$ years have clinically significant depression. ${ }^{11}$ Late-life depression (LLD) is associated with severe morbidities including weight loss, chronic medical illness, selfperception of poor health, functional impairment, and 
cognitive decline. ${ }^{12}$ In addition, LLD is associated with an elevated risk of suicide. ${ }^{13}$ In Hong Kong, $53 \%$ of suicidal older people have depression; depression is a major risk factor for late-life suicide. ${ }^{14}$

Compared with never-depressed individuals, people with depression are more deeply affected by negative life events and report a higher level of stress ${ }^{15}$ more negative mood, and less positive mood. ${ }^{16,17}$ Their emotional responses towards adverse events may be worsened by negative evaluation bias, lack of self-efficacy, and maladaptive patterns of coping that are characterised by depression. ${ }^{18,19}$ Individuals with depression have greater increases in negative appraisals after stressful events and a low level of coping efficacy in relieving distress,${ }^{20}$ which is associated with poor psychological adjustment outcome. ${ }^{21}$ During the SARS epidemic, older people developed deeper fears about the epidemic. They were more pessimistic, did not pay attention to positive information, and were overwhelmed by negative news. The feelings of less confidence in coping contributed to a higher tendency toward suicidal acts. ${ }^{6}$ Hence, patients with LLD are more vulnerable to adverse life events and more susceptible to suicidal risk during the COVID-19 pandemic.

It is crucial to provide early suicidal detection and intervention. Suicidal ideation is a significant risk factor and precursor for suicide attempts and completed suicides. ${ }^{22}$ It is important to assess levels of suicidal ideation to identify those who are at risk. We hypothesised that patients with LLD are at increased risk and should receive urgent attention. This study aims to compare patients with LLD and healthy controls in terms of suicidal ideation during the COVID-19 pandemic, and to determine predictors of suicidal ideation.

\section{Methods}

Between March and April 2020, old adults diagnosed with major depressive disorder (single or recurrent episode) as defined by the DSM-5 were recruited from psychiatric clinics or inpatient wards, whereas 31 healthy older adults without a history of depression or other psychiatric illnesses were recruited from voluntary organisations or elderly community centres. Their demographic characteristics (such as age, sex, and education level) were recorded. Medical comorbidity was assessed using the Cumulative Illness Rating Scale. ${ }^{23}$ Level of cognition was measured using the validated Hong Kong Chinese version of the Montreal Cognitive Assessment 5 minutes protocol (HKMoCA 5-mins protocol $)^{24}$ to exclude those with dementia. Depressive symptoms were assessed using the Hamilton Depression Rating Scale (HAM-D), ${ }^{25}$ which is a widely used and reliable measure of depressive symptoms. Scores range from 0 to 52; higher scores indicate more severe depression.

Participants were asked about their COVID-19-related experiences including: (1) whether they have contracted the disease, (2) whether they have been quarantined, (3) whether their friends or relatives have ever been quarantined or confirmed to have COVID-19; and (4) whether people they have close contact with have ever been quarantined or confirmed to have COVID-19. Perceived severity of the pandemic was assessed using the question: How would you rate the degree of severity regarding the COVID-19 pandemic?" Response options were 1: not very serious, 2: not serious, 3: fair, 4: serious, and 5: very serious. Perceived time spent on receiving related information was measured by the question: "How many hours on average do you spend to follow COVID-19-related information per day?

Perceived health was measured using the summed scores of two items (How is your present health? and How is your present physical functioning?) on a 5-point scale (1: extremely poor, 2: poor, 3: average, 4: good, and 5: excellent). Lower scores indicate poorer perceived health.

Level of loneliness was assessed using the 6-item De Jong Gierveld scale. ${ }^{26}$ It is a reliable and valid instrument for measuring overall, emotional, and social loneliness. Total scores range from 0 to 6 ; higher scores indicate a higher level of loneliness. The Chinese version of the scale has been validated in older adults in Hong Kong. ${ }^{27}$

Perceived coping efficacy was assessed using the COVID-19 coping efficacy inventory adapted from the SARS Appraisal Inventory. ${ }^{28}$ It measures physical health, facial and bodily appearances, daily life, family relationships, interpersonal relationships, religious beliefs, personal life goals, and value systems in a 5-point scale (0: none, 4: very great) to determine their confidence to cope with the impact. The internal consistency of the scale is satisfactory (alpha $=0.956$ ).

Suicidal ideation was assessed using the Geriatric Suicide Ideation Scale (GSIS). ${ }^{29}$ It consists of 31 items in a 5-point scale assessing suicide ideation, life orientation, loss of personal and social worth, and death ideation. Higher scores indicate higher levels of suicidal ideation. The Chinese version of GSIS has been validated in Hong Kong Chinese older adults. ${ }^{30}$

The 22-item self-report Impact of Event Scale-Revised $\left(\right.$ IES-R) ${ }^{31}$ measures the level of symptomatic responses to a specific traumatic stressor in the past week on a 5-point scale (0: absence of symptoms, 4: maximal symptoms). The Chinese version of the IES-R has good internal consistency and favourable scale equivalence with the original English version. ${ }^{32}$

Statistical analyses were performed using SPSS (Windows version 25; IBM Corp, Armonk [NY], US). All tests were two-tailed with the significance level set at $\mathrm{p}<0.05$. Chi-squared tests and $t$-tests were used to test between-group differences. If significant differences in depressive symptoms and other demographic variables were observed, a one-way between-group analysis of covariance was conducted to explore the impact of group on suicidal ideation and level of post-traumatic stress, with the group difference being controlled. Correlation analyses were used to examine bivariate relationships. The contribution of possible predictors was tested using multivariate linear 
regression after adjusting for depressive symptoms and other potential confounders. Possible mediation effects were further examined using bootstrapping procedures with the SPSS computational tool PROCESS. ${ }^{33}$

\section{Results}

In total, 21 men and 43 women aged 61 to 89 (mean, 72.8) years were interviewed through telephone by trained research assistants. Of them, 33 were old adults with LLD (cases) and 31 were healthy older adults (controls). The LLD group and healthy control group were comparable in terms of age $\left(\chi^{2}=22.6\right.$, df $\left.=22, p=0.427\right)$, sex $\left(\chi^{2}=0.195\right.$, $\mathrm{df}=1, \mathrm{p}=0.659)$, years of education $\left(\chi^{2}=15.6, \mathrm{df}=16\right.$, $\mathrm{p}=0.483)$, and COVID-19-related experience $\left(\chi^{2}=0.067\right.$, $\mathrm{df}=1, \mathrm{p}=0.796$ ) [Table 1]. The LLD group had a higher level of medical comorbidity $(\mathrm{t}(62)=6.41, \mathrm{p}<0.001)$, depressive symptoms $(\mathrm{t}(62)=2.55, \mathrm{p}=0.013)$, level of loneliness $(\mathrm{t}(62)=2.18, \mathrm{p}=0.033)$, and level of suicidal ideation $(\mathrm{t}(61)=4.40, \mathrm{p}<0.001)$ and a lower level of perceived coping efficacy $(\mathrm{t}(62)=-3.95, \mathrm{p}<0.001)$. There was no significant difference in the level of traumatic stress, amount of time spent on getting COVID-19-related information, perceived severity, perceived health, and cognition.
Aftercontrolling for the level of depression and medical comorbidity, analysis of covariance indicated a significant main effect for group across the GSIS scale $(F(1,59)=5.72$, $\mathrm{p}=0.020$ ). The LLD group had a significantly higher level of suicidal ideation than the healthy control group, even after controlling for the effect of depressive symptoms. There was no significant difference in the level of stress between groups across the IES-R scale $(F(1,60)=0.210$, $\mathrm{p}=0.649)$.

Coping efficacy negatively correlated with the GSIS score $(r=-7.06, \mathrm{p}<0.001)$ and IES-R score $(r=-3.49$ $\mathrm{p}=0.005)$, whereas loneliness positively correlated to the GSIS score $(r=0.566, \mathrm{p}<0.001)$ and IES-R score $(r=0.608$, $\mathrm{p}<0.001)$. A lower level of coping efficacy and a higher level of loneliness were associated with a higher level of suicidal ideation and traumatic stress.

To estimate the possible impacts of the predictors on outcome, multiple regression analyses were conducted (with the GSIS and IES-R scores as dependent variables). Depressive symptoms and medical comorbidity were controlled using a hierarchical regression model. The rating on the De Jong Gierveld scale and perceived coping efficacy were entered as independent variables. Overall, the amount of total variance in GSIS and IES-R scores accounted for by these variables was significant. The GSIS, HAM-D, and

Table 1. Clinical features of older people with late-life depression and healthy controls

\begin{tabular}{|lcc|}
\hline & $\begin{array}{c}\text { Older people with late-life } \\
\text { depression }(\mathbf{n}=\mathbf{3 3})^{*}\end{array}$ & Healthy controls $(\mathbf{n}=\mathbf{3 1})^{*}$ \\
Age, y & $74.45 \pm 6.41$ & $71.10 \pm 7.69$ \\
\hline Women & $23(70)$ & $20(65)$ \\
\hline Years of education & $6.71 \pm 4.86$ & $8.35 \pm 3.58$ \\
\hline COVID-19-related experience & 0 & 0 \\
$\quad$ Infected & $1(3)$ & 0 \\
$\quad$ Being quarantined & 0 & $4(13)$ \\
Friends or relatives being quarantined & $5(15)$ & 0 \\
Close contact being quarantined & $1.84 \pm 1.29$ & $1.47 \pm 1.39$ \\
\hline Hours receiving COVID-19 information per day & $4.36 \pm 0.78$ & $4.39 \pm 0.72$ \\
\hline Perceived COVID-19 severity & $3.32 \pm 0.72$ & $3.55 \pm 0.58$ \\
\hline Perceived health & $6.12 \pm 2.15$ & $2.68 \pm 2.15$ \\
\hline Cumulative Illness Rating Scale score & $24.75 \pm 3.80$ & $25.89 \pm 2.90$ \\
\hline Montreal Cognitive Assessment 5 minutes protocol score & $6.76 \pm 6.01$ & $3.45 \pm 4.11$ \\
\hline Hamilton Depression Rating Scale score & $3.18 \pm 1.70$ & $2.23 \pm 1.80$ \\
\hline De Jong Gierveld scale score & $18.27 \pm 5.69$ & $23.45 \pm 4.71$ \\
\hline Coping efficacy inventory score & $79.00 \pm 19.97$ & $58.94 \pm 15.90$ \\
\hline Geriatric Suicide Ideation Scale score & $13.21 \pm 10.80$ & $10.77 \pm 10.43$ \\
\hline Impact of Event Scale-Revised score & & \\
\hline
\end{tabular}

Data are presented as mean \pm standard deviation or No. (\%) of participants 
Cumulative Illness Rating Scale scores together accounted for $37.7 \%$ of the variance, and the model accounted for $60.6 \%$ of the variance after adding grouping, loneliness, and coping efficacy $(\mathrm{F}(3,57)=11.024, \mathrm{p}<0.001)$. However, only loneliness and coping efficacy were significant predictors (Table 2). Mediation analysis found a significant indirect effect of group on suicidal ideation through coping efficacy (indirect effect $=6.02$, standard error $=2.81,95 \%$ confidence interval $=1.41-12.06)$. Sobel test showed that coping efficacy mediated the relationship between group and suicidal ideation ( $\mathrm{Z}=2.43, \mathrm{p}=0.015$, Figure). For IES-R scoring, HAM-D alone accounted for $45.3 \%$ of the variance, and the model accounted for 52.3\%, after adding loneliness and coping efficacy $(\mathrm{F}(2,60)=4.451, \mathrm{p}=0.016)$. Only loneliness was a significant predictor in the model.

\section{Discussion}

The level of suicidal ideation was significantly higher in the LLD group than the healthy control group after adjusting for depressive symptoms. Older people with LLD had a significantly higher suicidal risk during the COVID19 pandemic and therefore early monitoring and timely intervention is needed.

Depression is associated with lower coping efficacy in stressful events, ${ }^{20}$ and low coping efficacy predicts poor adjustment outcomes in physical and psychological health. ${ }^{21}$ Coping efficacy (the confidence to cope with the impact of an event ${ }^{28}$ ) may partially explain the mechanism between grouping and level of suicidal ideation. The LLD group was associated with a lower level of coping efficacy toward

Table 2. Hierarchical regression analyses of the effects of coping efficacy and loneliness on suicidal ideation and stress

\begin{tabular}{|c|c|c|c|c|c|}
\hline & B & SE & $\beta$ & $\mathbf{t}$ & p Value \\
\hline \multicolumn{6}{|l|}{ Suicidal ideation } \\
\hline \multicolumn{6}{|l|}{ Step 1} \\
\hline Hamilton Depression Rating Scale & 1.73 & 0.43 & 0.455 & 4.013 & $<0.001$ \\
\hline Cumulative Illness Rating Scale & 1.93 & 0.84 & 0.26 & 2.28 & 0.026 \\
\hline \multicolumn{6}{|l|}{ Step 2} \\
\hline $\begin{array}{l}\text { Grouping (healthy controls }=0 \text {, older } \\
\text { people with late-life depression }=1 \text { ) }\end{array}$ & 3.60 & 4.74 & 0.09 & 0.76 & 0.451 \\
\hline Coping efficacy & -1.63 & 0.38 & -0.46 & -4.11 & $<0.001$ \\
\hline Loneliness & 2.78 & 1.27 & 0.24 & 2.19 & 0.033 \\
\hline \multicolumn{6}{|l|}{$R^{2}$ change $=0.229$} \\
\hline \multicolumn{6}{|l|}{ Stress } \\
\hline \multicolumn{6}{|l|}{ Step 1} \\
\hline Hamilton Depression Rating Scale & 1.32 & 0.19 & 0.67 & 7.16 & $<0.001$ \\
\hline \multicolumn{6}{|l|}{ Step 2} \\
\hline Coping efficacy & 0.27 & 0.21 & 0.15 & 1.30 & 0.20 \\
\hline Loneliness & 1.99 & 0.69 & 0.34 & 2.90 & 0.005 \\
\hline$R^{2}$ change $=0.071$ & & & & & \\
\hline
\end{tabular}

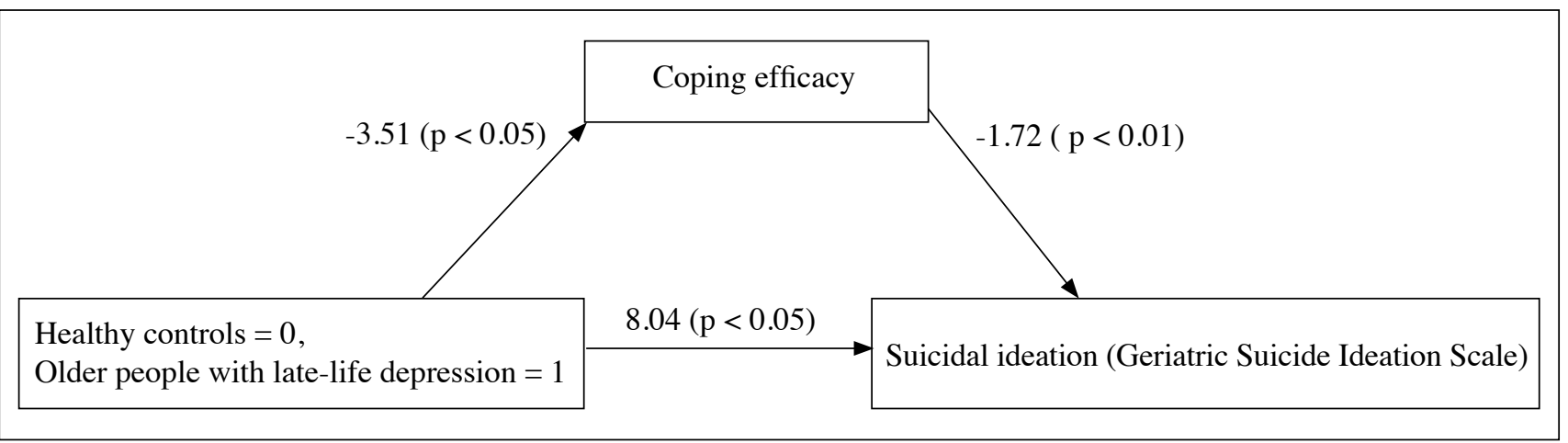

Figure. Path model with suicidal ideation as the outcome variable 
the COVID-19 pandemic, which was associated with a higher level of suicidal ideation. The appraisal process of an event plays a role in the development and maintenance of depression. ${ }^{34}$ Lower levels of health-related confidence are associated with suicide risk across different time points from suicidal ideation to future intention. ${ }^{35}$ Our findings provided empirical support to the link between coping efficacy and suicidal ideation in patients with LLD and to the mechanism of how suicidal ideation develops or maintains, with important implications for suicidal intervention programmes. Clinicians can teach patients with LLD-coping skills to boost their coping confidence and reinforce their perceived ability to cope with the pandemic.

Loneliness was associated with the level of suicidal ideation and the level of traumatic stress related to the COVID-19 pandemic. Individuals who experience suicidal ideation are often disconnected from others. ${ }^{36}$ Social isolation, entrapment, and loneliness contribute to suicide risk ${ }^{37}$ and are associated with suicidal thoughts and behaviours. ${ }^{36}$ Social isolation and loneliness during the pandemic are probably related to the quarantine and social distancing measures implemented. The feelings of social isolation and loneliness are probably more evident in those who live alone or have difficulty in using the internet and require different ways to stay in touch with others. ${ }^{2}$ Leading suicide theories emphasise the role of social connections in suicidal prevention, ${ }^{38}$ and thus reconnecting at-risk older people is crucial. Suicidal prevention programmes and community support should be provided through online, telephone, or other modalities to help those who are disconnected during the pandemic.

There was no significant difference between the LLD group and the healthy control group in terms of the level of traumatic stress. This may be due to the nature of the scale, which aims to capture the level of post-traumatic symptomatic responses of a stressful traumatic event. As the study was conducted during the pandemic, the level of acute stress may not be fully reflected. Nevertheless, as the pandemic is ongoing, the level of suicidal risk is expected to increase further should the stress level increase.

There are limitations to the study. Only 64 participants were recruited; the small sample size might have limited power to determine significant relationships between variables. Given the cross-sectional design, the causal relationships shown in this study remain tentative at best. Alternative paths of influence such as the effects of suicidal ideation and psychological distress on coping efficacy and loneliness are possible. Future research should investigate the predictive abilities of these variables using longitudinal designs. In addition, although participants were instructed to report their level of distress concerning COVID-19related experiences, the outcome may be affected by other life stressors that were not measured in our study. As none of the respondents had a personal experience related to the COVID-19 pandemic, the findings may not reflect the difference in suicidal risk and psychological distress between patients with LLD and healthy controls when facing traumatic experiences in a pandemic.

\section{Conclusion}

Early detection of suicidal risk in patients with LLD is important during a pandemic. Suicidal prevention measures should include enhancing the coping efficacy and skills of older people with depression to handle the unease situation and boosting their confidence to cope with the crisis. Mental health services and support programmes are crucial to reconnect vulnerable members and to promote an active and socially engaged lifestyle in older people.

\section{Funding}

This research received no specific grant from any funding agency, commercial or not-for-profit sectors

\section{Declaration}

All authors have no conflicts of interest to disclose.

\section{References}

1. Gunnell D, Appleby L, Arensman E, Hawton K, John A, Kapur N, et al. Suicide risk and prevention during the COVID-19 pandemic. Lancet Psychiatry 2020;7:468-71. Crossref

2. Yip PSF, Chau PH. Physical distancing and emotional closeness amidst COVID-19. Crisis 2020;41:153-5. Crossref

3. Yao H, Chen JH, Xu YF. Patients with mental health disorders in the COVID-19 epidemic. Lancet Psychiatry 2020;7:e21. Crossref

4. Chan SM, Chiu FK, Lam CW, Leung PY, Conwell Y. Elderly suicide and the 2003 SARS epidemic in Hong Kong. Int J Geriatr Psychiatry 2006;21:113-8. Crossref

5. Cheung YT, Chau PH, Yip PS. A revisit on older adults suicides and Severe Acute Respiratory Syndrome (SARS) epidemic in Hong Kong. Int J Geriatr Psychiatry 2008;23:1231-8. Crossref

6. Yip PS, Cheung YT, Chau PH, Law YW. The impact of epidemic outbreak: the case of severe acute respiratory syndrome (SARS) and suicide among older adults in Hong Kong. Crisis 2010;31:8692. Crossref

7. Taylor S. The Psychology of Pandemics: Preparing for the Next Global Outbreak of Infectious Disease. Cambridge Scholars Publishing; 2019.

8. Priya PK, Rajappa M, Kattimani S, Mohanraj PS, Revathy G. Association of neurotrophins, inflammation and stress with suicide risk in young adults. Clin Chim Acta 2016;457:41-5. Crossref

9. Qiu J, Shen B, Zhao M, Wang Z, Xie B, Xu Y. A nationwide survey of psychological distress among Chinese people in the COVID-19 epidemic: implications and policy recommendations. Gen Psychiatr 2020;33:e100213. Crossref

10. Asmundson GJG, Paluszek MM, Landry CA, Rachor GS, McKay D, Taylor S. Do pre-existing anxiety-related and mood disorders differentially impact COVID-19 stress responses and coping? J Anxiety Disord 2020;74:102271. Crossref

11. Chi I, Yip PS, Chiu HF, Chou KL, Chan KS, Kwan CW, et al. Prevalence of depression and its correlates in Hong Kong's Chinese older adults. Am J Geriatr Psychiatry 2005;13:409-16. Crossref

12. Blazer DG. Depression in late life: review and commentary. J Gerontol A Biol Sci Med Sci 2003;58:249-65. Crossref

13. Depression: Fact Sheet: World Health Organization; 2012. http://www. who. int/mediacentre/factsheets/fs369/en/index.

14. Chiu HF, Yip PS, Chi I, Chan S, Tsoh J, Kwan CW, et al. Elderly suicide in Hong Kong--a case-controlled psychological autopsy study. 
Acta Psychiatr Scand 2004;109:299-305. Crossref

15. Devanand DP, Kim MK, Paykina N, Sackeim HA. Adverse life events in elderly patients with major depression or dysthymic disorder and in healthy-control subjects. Am J Geriatr Psychiatry 2002;10:26574. Crossref

16. O'Grady MA, Tennen H, Armeli S. Depression history, depression vulnerability and the experience of everyday negative events. J Soc Clin Psychol 2010;29:949-74. Crossref

17. Conner TS, Tennen H, Zautra AJ, Affleck G, Armeli S, Fifield J. Coping with rheumatoid arthritis pain in daily life: within-person analyses reveal hidden vulnerability for the formerly depressed. Pain 2006;126:198-209. Crossref

18. Haaga D, Beck A, Paykel E. Handbook of Affective Disorders. 1992.

19. Abramson LY, Metalsky GI, Alloy LB. Hopelessness depression: a theory-based subtype of depression. Psychol Rev 1989;96:35872. Crossref

20. Gunthert KC, Cohen LH, Armeli S. Unique effects of depressive and anxious symptomatology on daily stress and coping. J Soc Clin Psychol 2005;21:583-609. Crossref

21. Lazarus RS. Stress and Emotion: A New Synthesis: Springer Publishing Company; 2006.

22. Mann JJ, Apter A, Bertolote J, Beautrais A, Currier D, Haas A, et al. Suicide prevention strategies: a systematic review. JAMA 2005;294:2064-74. Crossref

23. Conwell Y, Forbes NT, Cox C, Caine ED. Validation of a measure of physical illness burden at autopsy: the Cumulative Illness Rating Scale. J Am Geriatr Soc 1993;41:38-41. Crossref

24. Wong A, Nyenhuis D, Black SE, Law LS, Lo ES, Kwan PW, et al. Montreal Cognitive Assessment 5-minute protocol is a brief, valid, reliable, and feasible cognitive screen for telephone administration. Stroke 2015;46:1059-64. Crossref

25. Zheng YP, Zhao JP, Phillips M, Liu JB, Cai MF, Sun SQ, et al. Validity and reliability of the Chinese Hamilton Depression Rating Scale. Br J Psychiatry 1988;152:660-4. Crossref

26. de Jong Gierveld J, van Tilburg T. A 6-item scale for overall, emotional, and social loneliness: confirmatory tests on survey data. Res Aging
2006;28:582-98. Crossref

27. Leung GT, de Jong Gierveld J, Lam LC. Validation of the Chinese translation of the 6-item De Jong Gierveld Loneliness Scale in elderly Chinese. Int Psychogeriatr 2008;20:1262-72. Crossref

28. Cheng SKW, Chong GHC, Chang SSY, Wong CW, Wong CSY, Wong MTP, et al. Adjustment to severe acute respiratory syndrome (SARS): roles of appraisal and post-traumatic growth. Psychol Health 2006;21:301-17. Crossref

29. Heisel MJ, Flett GL. The development and initial validation of the geriatric suicide ideation scale. Am J Geriatr Psychiatry 2006;14:74251. Crossref

30. Chou KL, Jun LW, Chi I. Assessing Chinese older adults' suicidal ideation: Chinese version of the Geriatric Suicide Ideation Scale. Aging Ment Health 2005;9:167-71. Crossref

31. Weiss DS, Marmar CR. The Impact of Events Scale-Revised. In: Wilson JP, Keane TM, editors. Assessing Psychological Trauma and PTSD. The Guilford Press; 1997: 399-411. Crossref

32. Wu KK, Chan KS. The development of the Chinese version of Impact of Event Scale--Revised (CIES-R). Soc Psychiatry Psychiatr Epidemiol 2003;38:94-8. Crossref

33. Hayes AF. Introduction to Mediation, Moderation, and Conditional Process Analysis: A Regression-Based Approach: Guilford publications; 2017.

34. Barlow DH. Clinical Handbook of Psychological Disorders, 5th ed: a Step-By-Step Treatment Manual. Guilford Publications; 2014.

35. Isaac V, Wu CY, McLachlan CS, Lee MB. Associations between health-related self-efficacy and suicidality. BMC Psychiatry 2018;18:126. Crossref

36. Van Orden KA, Witte TK, Cukrowicz KC, Braithwaite SR, Selby EA, Joiner TE Jr. The interpersonal theory of suicide. Psychol Rev 2010;117:575-600. Crossref

37. O'Connor RC, Kirtley OJ. The integrated motivational-volitional model of suicidal behaviour. Philos Trans R Soc Lond B Biol Sci 2018;373:20170268. Crossref

38. Reger MA, Stanley IH, Joiner TE. Suicide mortality and coronavirus disease 2019: a perfect storm? JAMA Psychiatry 2020. Crossref 